\title{
SELETIVIDADE DE INSETICIDAS USADOS NA CULTURA DO MILHO PARA ADULTOS DE DORU LINEARE (ESCHSCHOLTZ, 1822) (DERMAPTERA: FORFICULIDAE)
}

\section{M.J. Zotti' ${ }^{1 *}$, A.D. Grützmacher ${ }^{1}$, D.D. Grützmacher ${ }^{1}$, G.O. Dalmazzo ${ }^{1 * *}$, J.F.S. Martins ${ }^{2}$}

'Universidade Federal de Pelotas , Faculdade de Agronomia “Eliseu Maciel”, Departamento de Fitossanidade, CP 354, CEP 96010-900, Pelotas, RS, Brasil. E-mail: mzotti.faem@ufpel.tche.br

\section{RESUMO}

\begin{abstract}
O objetivo do presente trabalho foi avaliar a seletividade dos inseticidas utilizados na cultura do milho sobre Doru lineare. Foi avaliada a seletividade de inseticidas utilizados na cultura do milho para $D$. lineare, seguindo metodologia proposta pela IOBC/WPRS em condições de laboratório. Adultos foram expostos aos resíduos dos inseticidas por meio de contato com placas de vidros e pelo consumo de ovos de Spodoptera frugiperda contaminados com os inseticidas. A porcentagem de mortalidade dos insetos em contato residual foi utilizada para classificar os inseticidas como inócuo (<30\%), levemente nocivo (30-79\%), moderadamente nocivo (80-99\%) e nocivo (>99\%). A mortalidade de adultos após consumo de ovos contaminados foi comparada com a testemunha. Para adultos de D. lineare mediante contato residual, nas 360 HAIE (horas após o início da exposição), os inseticidas Decis 25 EC, Dimilin, Engeo Pleno, Karate Zeon 250 CS, Lorsban 480 BR, Match EC, Neem Azal eSevin 480SC foram nocivos ao predador. Certero, Fastac 100SC e Tracer foram moderadamente nocivos. Intrepid 240 SC e Mimic 240 SC foram inócuos. A mortalidade de adultos após o consumo de ovos contaminados foi significativa para os inseticidas Certero, Dimilin, Tracer, Engeo Pleno, Lorsban 480 BR, Sevin 480 SC.
\end{abstract}

PALAVRAS-CHAVE: Controle biológico, controle químico, manejo integrado de pragas, toxicidade, Zea mays.

\begin{abstract}
SELECTIVITY OF INSECTICIDES USED INTHE CORN CROPTO ADULTSOFDORULINEARE (ESCHSCHOLTZ, 1822) (DERMAPTERA: FORFICULIDAE). The objective of this work was to evaluate the selectivity of insecticides used in the corn crop to Doru lineare (Eschscholtz, 1822) (Dermaptera:Forficulidae), following methodology suggested by IOBC/WPRS, under laboratory conditions. Adult insects were placed on glass plates or fed on Spodoptera frugiperda eggs, both containing previously sprayed insecticide residues. The adult mortality by the residual contact was used to classify insecticides as harmless (<30\%), slightly harmful (30-79\%), moderately harmful (80-99\%) and harmful (>99\%). The adult mortality due to consumption of the contaminated eggs was compared to the control. For adults of $D$. linearein residual contact after 360 HASE (hours after start of exposure) the insecticides Decis 25 EC, Dimilin, Engeo Pleno, Karate Zeon 250 CS, Lorsban 480 BR, Match EC, Neem Azal and Sevin 480 SC were harmful. Certero, Fastac 100 SC and Tracer were moderately harmful. Intrepid 240 SC and Mimic 240 SC were slightly harmful. The mortality of adults after consumption of contaminated eggs was noteworthy for the insecticides Certero, Dimilin, Tracer, Engeo Pleno, Lorsban 480 BR and Sevin 480 SC.
\end{abstract}

KEY WORDS: Biological control, chemical control, integrated pest management, toxicity,Zeamays.

\section{INTRODUÇÃO}

Em agroecossistema de várzea a ação de insetospraga, sem dúvida, é um dos principais fatores que afeta a economicidade das lavouras de milho, por impedir o melhor aproveitamento do potencial produtivo dos híbridos atualmente disponíveis (BUSATO et al., 2006), sendo que a lagarta Spodoptera frugiperda

\footnotetext{
${ }^{2}$ Embrapa Clima Temperado, Pelotas, RS, Brasil.

*Parte da Dissertação de Mestrado do primeiro autor, Programa de Pós-Graduação em Fitossanidade. Apoio: bolsa de estudos da Coordenação de Aperfeiçoamento de Pessoal de Nível Superior (CAPES).

${ }^{* *}$ Acadêmico Agronomia, PIBIC CNPq, Depto. de Fitossanidade-FAEM-UFPel.
} 
(J.E.Smith,1797) (Lepidoptera: Noctuidae) é considerada uma das principais pragas, ocasionando altos índices de desfolhamento do milho nestas condições (GRÜTZMACHER et al., 2000).

Em várzea, o controle dessa praga tem sido feito com inseticidas químicos, geralmente não seletivos aos inimigos naturais. Os abusos nas aplicações desses inseticidas produzem, na maioria das vezes, uma redução no número de artrópodes benéficos, quando comparados com outras práticas agrícolas. Desta forma, nos últimos anos últimos, tem-se dado preferência por produtos químicos eficientes, porém, também seletivos, principalmente aos inimigos naturais de maior ocorrência no agroecossistema, de forma a preservá-los e dar um controle mais duradouro das pragas na cultura do milho (Zотті et al., 2008).

Na cultura do milho a espécie Doru lineare (Eschscholtz, 1822) (Dermaptera: Forficulidae) é encontrada predando ovos e lagartas de vários tamanhos de $S$. frugiperda (ReIs et al., 1988) sendo a média de ovos e lagartas predados entre machos efêmeas da tesourinha, de 47,5 e 43,2 por dia, respectivamente (SASAKi et al., 1986). Além disso, D. lineare, em áreas de várzea localizada na região sul do Estado do Rio Grande do Sul, destaca-se como um dos principais predadores de pragas, especialmente na cultura do milho (Zотті et al., 2008).

Segundo a "International Organization for Biological and Integrated Control of Noxious Animals and Plants (IOBC), West Paleartic Regional Section (WPRS)", a estratégia de manejo integrado deve incluir estudos de seletividade de pesticidas para inimigos naturais, avaliando-se os efeitos sobre a fauna global de uma cultura ou sobre uma determinada espécie, baseando-se numa sequência de teses de toxicidade, iniciando-se em laboratório e continuando em condições de semicampo e campo (Hassan etal., 1985).

A seletividade deinseticidas utilizados na cultura do milho para adultos deDoru luteipes (Scudder,1876) (Dermaptera: Forficulidae) foi estudada porFALEIROet al.(1995), os quais concluíram que esse inseto foi mais tolerante aos inseticidas quando na fase adulta em comparação com o estágio ninfal, e que o inseticida mais seletivo foi permetrina 500 CE seguida da deltametrina 25 CE. De forma similar, PICANÇO et al. (2003), avaliando inseticidas usados para o controle de Ascia monustes orseis (Godart, 1818) (Lepidoptera: Pieridae), observaram que permetrina $500 \mathrm{CE}$ e deltametrina 25 EC foram altamente seletivos para ninfas e adultos de $D$. luteipes.

Em literatura é comum trabalhos com insetos do gênero Doru principalmente com a espécie $D$.luteipes, o que não ocorre para $D$. lineare. Sendo assim, o objetivo do presente trabalho foi avaliar a seletividade de inseticidas usados para o controle de S. frugiperda na cultura do milho, para adultos do predador $D$. lineare.

\section{MATERIAL E MÉTODOS}

Os experimentos foram conduzidos no Laboratório do Núcleo de Manejo Integrado de Pragas (NUMIP), Embrapa Clima Temperado/UFPel, Capão do Leão, RS.

Foram coletados ovos, ninfas e adultos de $D$. lineare na cultura do milho no agroecossistema de várzea subtropical no Município de Pelotas, RS. Em laboratório, os insetos foram mantidos em temperatura de 25 $\pm 1^{\circ} \mathrm{C}$, umidade relativa $70 \pm 10 \%$ e fotofase 14 horas, dentro de caixas de vidro com 30 centímetros de cada lado. Para evitar a fuga dos insetos foi aplicado na parte superior de cada gaiola talco farmacêutico inodoro. Os insetos foram alimentados com uma mistura de farinha de pupa de insetos e pólen comercial, seguindo a técnica de criação adaptada, descrita por PAsini et al. (2007).

Para os trabalhos de contato residual foram utilizados 13 inseticidas pertencentes a diferentes grupos químicos (Tabela 1). Os inseticidas testados são geralmente usados na cultura do milho, à exceção de Neem Azal (Trifolio M GmbH; Lahnau, Alemanha). Os inseticidas foram aplicados por meio de pulverizador pressurizado a $\mathrm{CO}_{2}$, utilizando-se bico tipo TeeJet TP80015E. A pressão de trabalho foi de 50 psi obtendo-se um depósito para cada composto de 1,75 $\pm 0,25$ mg. $\mathrm{cm}^{-2}$, conforme metodologia da IOBC/WPRS (HASsAn et al., 1985).

As aplicações foram realizadas em placas de vidro com dimensões de $42 \mathrm{~cm} \times 22 \mathrm{~cm} \times 0,3 \mathrm{~cm}$, as quais, após secar o excesso de umidade de suas superfícies, foram cobertas por outra placa de acrílico de mesmo tamanho mas que possuíam dez orifícios, nos quais foram colocadas arenas de vidro, com $4 \mathrm{~cm}$ de altura e $5 \mathrm{~cm}$ de diâmetro, vazada em ambos os lados. No interior das arenas foi aplicado talco farmacêutico como descrito anteriormente.

Adultosdopredadorcom três diasdeidadeeobtidos dacriação delaboratório foram liberadosnointerior das arenas, ficandoem contatoconstantecomosinseticidas em teste, durante todo o período de avaliação. As mortalidades acumuladas dos insetos foram avaliadas em períodos de 24 horas durante quinze dias, ou seja, 360 horas após o início da exposição (HAIE). Cada tratamento foi composto por três repetições com dez insetos cada. Foram utilizadas duas testemunhas (Tabela 2), sendo a testemunha positiva previamente obtida por screening econsiderada como padrão de toxicidade para D. lineare. Para a testemunha negativa foram utilizadas placas pulverizadas comágua. O delineamento utilizado foi de blocos ao acaso. 
Com base nas porcentagens de mortalidade corrigidas pela fórmula de Abbott (Аввотт, 1925), os inseticidas foram classificados segundo índices propostos pela IOBC/WPRS, em: 1) inócuo $(<30 \%) ; 2)$ levemente nocivo $(30-79 \%)$; 3 ) moderadamente nocivo $(80-99 \%)$ e 4$)$ nocivo (> $99 \%)$.

Em outro experimento, foi avaliado o efeito da ingestão de ovos de $S$. frugiperda com resíduos dos inseticidas. As aplicações dos inseticidas (Tabela 1) sobre ovos desse noctuídeo foram realizadas por meio de torre de Potter calibrada à pressão de 10 psi resultando num volume de 1,7 $\pm 0,205 \mathrm{mg} . \mathrm{cm}^{-2}$, seguindoa metodologia proposta por SIMÕEs et al. (1998). Imediatamente após as aplicações dos inseticidas, os ovos de $S$. frugiperda foram ofertados aos adultos de $D$. lineare, que foram acondicionados em placas de Petri com $9 \mathrm{~cm}$ de diâmetro. As placas foram cobertas com filme plástico de $\mathrm{PVC}$, sendo que foram feitos pequenos orifícios, permitindo desta forma a ventilação. Avaliou-se a mortalidade dos insetos às 120 horas após a ingestão dos ovos tratados com os inseticidas. Esse período foi determinado, pois, durante as avaliações, verificou-se que alguns insetos após ingerirem os ovos contaminados com piretroides ou Neem Azal intoxicaram-se, mas não morreram. Cada placa de Petri com quatro adultos de $D$. lineare foi considerada uma repetição, num total de quatro repetições. O delineamento utilizado foi de blocos ao acaso.

Os dados de todos os experimentos foram submetidos à análise de variância, sendo que as médias foram comparadas por meio do teste de agrupamento de Scott-Knott a 5\% de significância (ScotT; KNotT, 1974), utilizando o programa Sisvar (FERREIRA, 2000).

\section{RESULTADOS E DISCUSSÃO}

Com relação à toxicidade causada pelos inseticidas piretroides ao predador D. lineare, Decis 25 EC (deltametrina) não provocou mortalidade significativa até 72 horas após o início da exposição (HAIE), apresentando baixa toxicidade quando comparado com os demais grupos químicos estudados e com os demais piretroides (Tabela 2). Constatou-se que, com o aumento do período de exposição, ocorreu maior mortalidade dos insetos e os grupos químicos apresentaram pequenas diferenças entre si, sendo este efeito menos evidente para Decis $25 \mathrm{EC}$, pois até 72 HAIE causou mortalidade semelhanteà testemunha, mas diferente dos outros piretroides (Tabela 2). Até 96 HAIE Decis 25 EC foi considerado inócuo (classe 1), enquanto Karate Zeon 250 CS (lambda-cialotrina) e Fastac 100SC (alfa-cipermetrina) foram enquadrados nas classes 2 e 3, respectivamente (Tabela 3). Neste sentido, Decis 25 EC apresentou baixa toxicidade inicial (poder de choque) em comparação com os demais piretroides (Tabela 3). Às 168 e 360 HAIE, todos os inseticidas piretroides causaram mortalidade semelhante à testemunha positiva, o inseticida Sevin 480SC (carbaril), sendo enquadrados na classe 4, entretanto, Fastac 100 SC foi considerado classe 3 (Tabela 3).

Tabela 1 - Inseticidas avaliados nos testes de seletividade para Doru lineare utilizando-se a dosagem máxima para o controle de Spodoptera frugiperda em milho.

\begin{tabular}{llllll}
\hline Produto comercial & Ingrediente ativo & Grupo químico & DC $^{1}$ & C.i.a $^{2}$ & C.p.c. $^{3}$ \\
\hline Certero & triflumurom & Benzoilureia & 0,05 & 0,0120 & 0,025 \\
Decis 25 EC & deltametrina & Piretroide & 0,20 & 0,0025 & 0,010 \\
Dimilin & diflubenzuron & Benzoiluréia & 0,10 & 0,0125 & 0,050 \\
Engeo Pleno & lambda-cialotrina + & Piretroide + & 0,25 & $0,013+$ & 0,0125 \\
& tiametoxam & Neonicotinoide & & 0,017 & \\
Fastac 100 SC & alfa-cipermetrina & Piretroide & 0,05 & 0,0025 & 0,025 \\
Intrepid 240 SC & metoxifenozida & Diacilhidrazina & 0,18 & 0,0216 & 0,090 \\
Karate Zeon 250 CS & lambda-cialotrina & Piretroide & 0,10 & 0,0125 & 0,050 \\
Lorsban 480 BR & clorpirifós & Organofosforado & 0,60 & 0,1440 & 0,300 \\
Match EC & lufenurom & Benzoilureia & 0,30 & 0,0075 & 0,150 \\
Mimic 240 SC & tebufenozida & Diacilhidrazina & 0,30 & 0,0360 & 0,150 \\
Neem Azal4 & azadirachtin & Azadiracthin & 1,00 & 0,5000 & 0,500 \\
Sevin 480 SC5 & carbaril & Carbamato & 0,36 & 0,0864 & 0,180 \\
Tracer & espinosade & Espinosinas & 0,10 & 0,0240 & 0,050 \\
\hline
\end{tabular}

${ }^{1} \mathrm{DC}=$ Dosagem de campo $\left(\mathrm{L} \mathrm{ou} \mathrm{kg} \mathrm{ha}{ }^{-1}\right.$ do produto comercial) considerando um volume de calda de $200 \mathrm{~L} \mathrm{ha}^{-1}$;

${ }^{2}$ C.i.a. = Concentração (\%) do ingrediente ativo na calda utilizada nos bioensaios;

${ }^{3}$ C.p.c. $=$ Concentração $(\%)$ do produto comercial na calda utilizada nos bioensaios;

${ }^{4}$ Produto não recomendado para o controle de Spodoptera frugiperda em milho;

${ }^{5}$ Testemunha positiva, inseticida reconhecidamente nocivo pela IOBC/WPRS, usado como padrão de toxicidade e obtido mediante screening. 


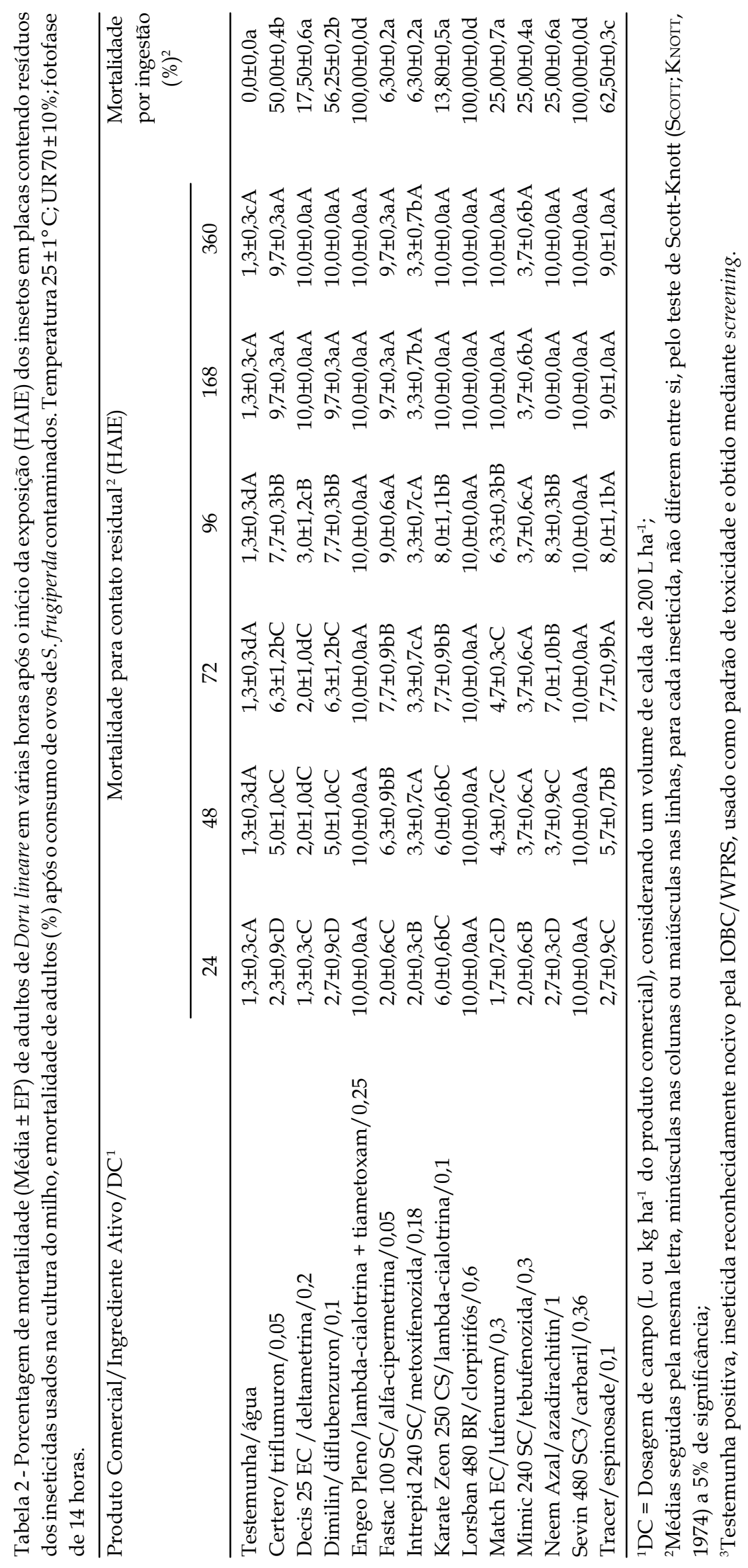


Seletividade de inseticidas usados na cultura do milho para

adultos de Doru lineare (Eschscholtz, 1822) (Dermaptera: Forficulidae).

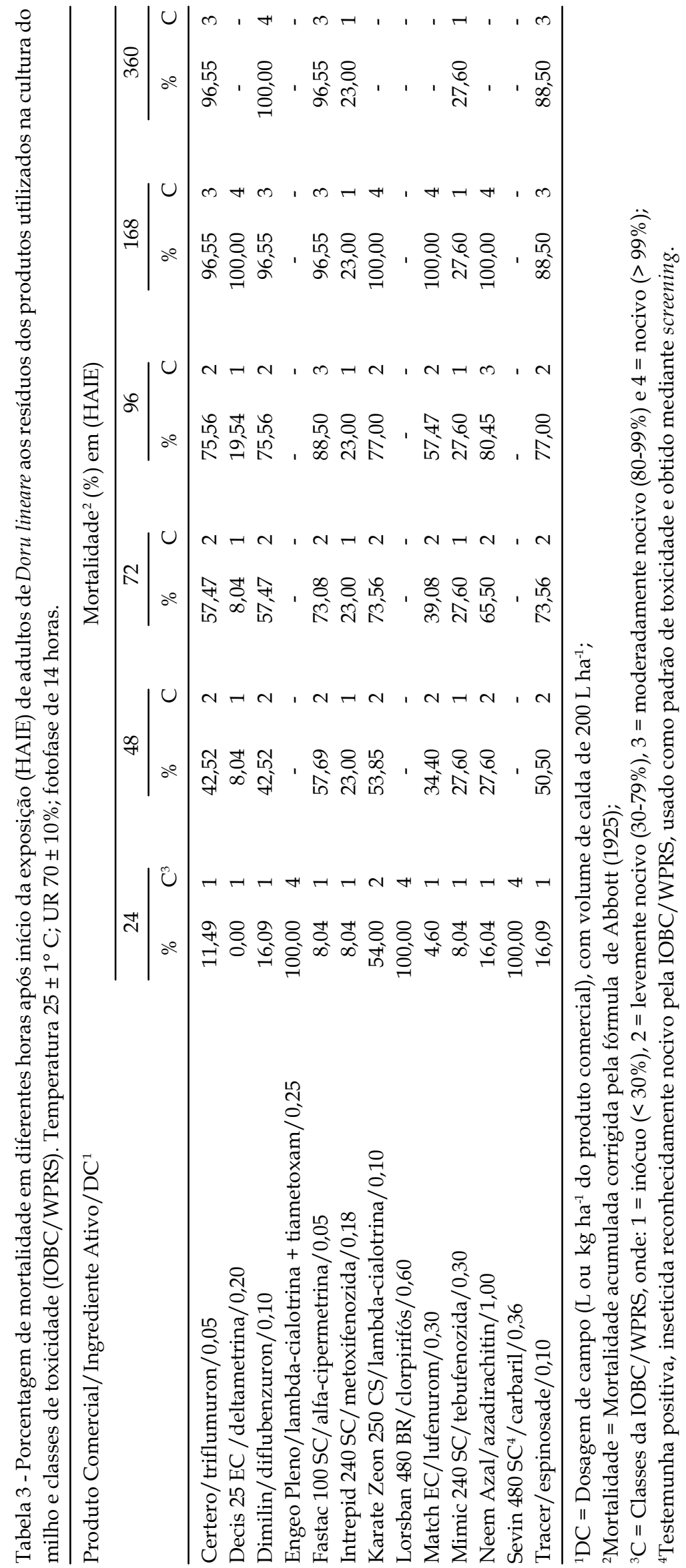


A seletividade de Decis 25 EC nas 24 HAIE paraD. luteipes também foi observada por ReIs et al. (1988) e Picanço et al. (2003) com mortalidades de 0,0 e 2,5\%, respectivamente, de forma semelhante aos resultados encontrados no presente estudo (Tabela 3). FALEIRO et al. (1995) obtiveram valores de mortalidades para adultos de $D$.luteipes de $2,4 \mathrm{e} 12,7 \%$ para os inseticidas piretroides Pounce $384 \mathrm{CE}$ (permetrina) e Decis 25EC (deltametrina), respectivamente. As diferenças de resultados em comparação aqueles encontrados no presente estudo (Tabela 3 ) podem estar relacionadas com a metodologia de teste usada, visto que nesses trabalhos os autores expuseram os insetos em contato com folhas de milho mergulhadas em calda com os inseticidas, e as avaliações foram realizadas após 24 horas da aplicação dos inseticidas.

Verificou-sequeaté as 96 HAIE ocorreu um aumento gradual na mortalidade dos insetos para os inseticidas piretroides Karate Zeon 250 CS e Fastac 100 SC e consequentemente nas classes de toxicidade no entanto, Decis 25ECneste período foi enquadrado na mesma classe (classe1). Esta maior seletividade de Decis 25EC (deltametrina) em favor de adultos de $D$. lineare pode estar relacionada ao seu elevado peso molecular $(505,2)$ quando comparados com aqueles do Karate Zeon 250, lambda-cialotrina $(391,3)$ e Fastac 100 SC, alfacipermetrina $(416,0)$, visto que substâncias de maior peso molecular possuem menor taxa de penetraçãona cutícula dos insetos (Sтоск; Holloway, 1993).

Verificou-se que todos os piretroides apresentaram diferença em relação à testemunha positiva (Sevin 480 SC) quando o predador foi alimentado com ovos de S. frugiperda contaminados; entretanto, foram semelhantes à testemunha (água), confirmando resultados observados porSimões etal.(1998), coma espécie D. luteipes, demonstrando desta forma a seletividade destes inseticidas piretroides avaliados via ingestão (Tabela 2).

Para os inseticidas inibidores da síntese dequitina Certero(triflumuron), Dimilin(diflubenzuron) eMatch EC (lufenurom) não foram encontradas diferenças estatísticas entre si nas mortalidades de adultos até 48 HAIE, bem como com as diacilhidrazinas, porém todos foram significativamente diferentes dos demais grupos químicos e de ambas as testemunhas. A partir das 168 HAIE, os benzoilureias foram tóxicos tanto quanto a testemunha positiva (Tabela 2), entretanto, foram enquadradosem diferentes classes de toxicidade (Tabela3). Referente às classes propostas pela IOBC/ WPRS, os benzoilureias foram classificados como inócuos (classe 1), na avaliação em 24 HAIE. Às 360 HAIE o inseticida Certero (triflumurom) foi classificadocomo moderadamente nocivo (classe 3 ), sendo que os demais benzoilureias causaram mortalidade de $100,00 \%$, sendo classificados como nocivos (classe 4 ) (Tabela 3).
VARGAS et al. (2004) estudaram o efeito de outros inibidores de síntese de quitina como Atabron 50 EC (clorfluazurom), Match EC (lufenurom) e Rimon 100 EC (novalurom) para adultos de D. luteipes 24 HAIE eencontraram médias de mortalidade semelhantes às observadas no presente estudo. Este fato pode ser explicado, pois a maioria dos inseticidas do grupo químico das benzoilureias não tem ação de choque, agindo principalmente por ingestão, no entanto em alguns insetos pode agir por contato (FRANÇA;BRANCO, 1996).

De acordo com RetNAKARAM; WRIGTH (1978), as benzoilureias atuam principalmente como ovicidase larvicidas, no entanto, efeitos sobre a longevidade e fertilidade de adultos também têm sido reportados. Por ingestão de ovos contaminados, os produtos Certero, Dimilin e Match EC causaram 50,00; 56,25 e $25,00 \%$ de mortalidade, respectivamente. Assim, é possível que estes produtos atuem na regeneração da membrana peritrófica em $D$. lineare, pois, segundo SOLTANI (1984), esta estrutura tem característica quitinosa, sendo afetada quando ocorre a ingestão de benzoilureias.

Intrepid 240 SC (metoxifenozida), Mimic 240 SC (tebufenozida) e Neem Azal (azadirachtin) não provocaram mortalidade significativa nas 24 HAIE (Tabela 2). Os inseticidas Intrepid 240 SC e Mimic 240 SC mantiveram a média de mortalidade dos insetos estável após a segunda avaliação (48 HAIE), não apresentando diferença entre si. Desta forma e, apesardas suas mortalidades serem diferentes da testemunha, esses inseticidas demonstraram baixa toxicidade aos insetos ao longo das avaliações (Tabela 2). Considerando as classes de toxicidade propostas pela IOBC/WPRS verificou-se que Neem Azal foi nocivo (classe 4) nas últimas duas avaliações. Os inseticidas Intrepid 240 SC e Mimic 240 SC foram classificados como inócuos (classe1) (Tabela3), sendo os únicos inseticidas, dentre todos os testados, que foram enquadrados nesta classe em todas as avaliações. Resultados semelhantes para esses dois inseticidas foram encontrados porMedinaet al.(2002) com o predador Chrysoperla carnea (Stephens, 1836) (Neuroptera: Chrysopidae), visto que não apresentaram efeito tóxico após consumirem ovos de $S$. frugiperda contaminados com os inseticidas. Deforma similar, CARVALHO et al. (2003) observaram que o inseticida Mimic 240SC não causou mortalidade de larvas de Chrysoperla externa (Hagen, 1861) (Neuroptera: Chrysopidae) após ingerirem ovos de Anagasta kuehniella (Zeller, 1879) (Lepidoptera: Pyralidae) contaminados com o inseticida.

A reduzida penetração das diacilhidrazinas em insetos adultos (26\%) explicaria em parte a tolerância dos insetos a estes produtos, via contato residual (Medina et al., 2002). Outra hipótese seria que as glândulas protorácicas, responsáveis pela produção 
dos ecdisteroides, degeneram no estágio adulto (RETNAKARAm et al., 1995), e desta forma, mesmo que uma pequena quantidade do produto penetrasse no corpo do inseto, não teria ação, visto a inexistência do seu principal sítio de atuação. Está hipótese também pode ser usada para explicar a baixa mortalidade dos insetos adultos contaminados via ingestão (Tabela 2).

Neem Azal prejudica a utilização dos nutrientes ingeridos, reduzindo a eficiência de conversão de alimento e a atividade das enzimas do mesêntero, ou intestino médio, além de afetar diretamente as células dos músculos do canal alimentar, diminuindo a frequência de contrações e aumentando a flacidez muscular, e ainda pode atuar nas mitocôndrias deixando-as inoperantes (TANZUBIL; MCCAFFERY, 1990). A espécie $D$. lineare, em função do seu comportamento, realiza uma constante limpeza do corpo pelas peças bucais (LANGSTON; POWELL, 1975) e, consequentemente, contamina-secom oresíduo deinseticida, contribuindo para as altas mortalidades por contato (Tabela 2).

Os inseticidas Lorsban 480 BR (clorpirifós) eSevin 480 SC (carbaril) (Tabela 2), causaram as mais altas médias de mortalidade de insetos adultos após ingerirem ovos de $S$. frugiperda contaminados, concordando com os encontrados por CARVALHO et al. (2003), os quais ofertaram ovos de A. kuehniella contaminados com o inseticida Vexter 480 CE (clorpirifós) para larvas de C. externa. Para contato residual, ambos inseticidas causaram mortalidade de $100 \%$ em 24 HAIE sendo enquadrados na classe 4 (Tabela 3). Resultados semelhantes foram obtidos com D. luteipes para Sevin 480 SC por FALEIRO et al. (1995) e PICANÇO et al. (2003). Reis et al. (1988) também observaram elevada mortalidade de $D$. luteipes por inseticidas organofosforados (Lorsban 15G e Lorsban 480 BR) e carbamatos (Lannate 5G e Furadan 5G).

As altas mortalidades $(100 \%)$, causadas por Lorsban 480 BR e Sevin 480 SC aos adultos do predador por contato e ingestão, possivelmente estejam relacionadas com o baixo peso molecular destes inseticidas (330,59 e 201,22; respectivamente) quando comparados aqueles dos piretroides, pois, segundo STоск; Holloway (1993), substâncias com pesos moleculares maiores possuem menor capacidade de penetração na cutícula do inseto.

A mortalidade de adultos de D. lineare referente ao consumo de ovos de $S$. frugiperda contaminados com Tracer (espinosade) foi de 62,5\% (Tabela 2), ocupando uma posição intermediária entre piretroides, benzoilureias, carbamatos e organofosforados. Cisneros et al. (2002) estudaram o efeito do inseticida Tracer sobre Doru taeniatum (Dohrn, 1847) (Dermaptera: Forficulidae), sendo que as mortalidades foram de $48 \%$ e $98 \%$ quando os insetos foram alimentados com grânulos contendo 1,2 ppm e 1200 ppm, respectivamente.
O inseticida Tracer na avaliação de 24 HAIE não provocou mortalidade dos insetos por contato (Tabela 2), sendo categorizado como inócuo, classe 1 (Tabela3). Às 168HAIEfoi semelhante ao inseticida padrão de toxicidade Sevin 480 SC. Nas últimas duas avaliações foi moderadamente nocivo (classe3) (Tabela 3).

$\mathrm{O}$ inseticida Engeo Pleno (lambda-cialotrina + tiametoxam) causou $100 \%$ de mortalidade de adultos de D. lineare às $24 \mathrm{HAIE}$ (Tabela 2), de forma semelhante ao inseticida padrão de toxicidade (Sevin 480 SC), demonstrado a sua alta toxicidade inicial. Da mesma forma, quando os insetos ingeriram ovos de $S$. frugiperda contaminados com esse inseticida, apresentaram $100 \%$ de mortalidade, sendo semelhante ao inseticida Sevin 480 (Tabela 2).

Os estudos de laboratório geralmente fornecem informações rápidas a respeito da seletividade dos pesticidas a várias espécies de inimigos naturais. Nessa condição, o inseto é submetido a mais alta forma de exposição e para aqueles produtos que se mostrarem inócuos não há necessidade de realização de testes em condições de semicampo e campo. Desta forma, estudos de seletividade em laboratório são necessários para separar os inseticidas inócuos dos demais, diminuindo o número de compostos que deverão ser testados em outras condições.

\section{CONCLUSÕES}

Às 360 horas após a exposição de $D$. lineare aos compostos Decis 25EC (deltametrina/0,0025), Dimilin (diflubenzuron/0,0125), Engeo Pleno (lambdacialotrina + tiametoxam/ 0,0130 + 0,0170), Karate Zeon 250 CS (lambda-cialotrina/0,0125), Lorsban 480 BR (clorpirifós/0,1440), Match EC (lufenurom/ 0,0075), Neem Azal (azadiracthin/0,5000) e Sevin 480 SC (carbaril/0,0864) foram nocivos (classe 4). Os inseticidas Certero (triflumuron/0,0120), Fastac 100 SC (alfa-cipermetrina/0,0025) e Tracer (espinosade/ 0,0240 ) foram moderadamente nocivos (classe 3), e Intrepid 240SC (metoxifenozida/0,0216) e Mimic 240 SC (tebufenozida/0,0360) foram inócuos (classe 1).

Certero, Dimilin, Tracer, Engeo Pleno, Lorsban 480 $\mathrm{BR}$, Sevin 480SC foram nocivos aos adultos deD.lineare que ingeriram ovos de $S$. frugiperda contaminados.

\section{REFERENCIAS}

ABBOTT, W.S. A method of computing the effectiveness of an insecticide. Journal of Economic Entomology, v.18, n.2, p.265-267, 1925.

BUSATO, G.R.; GRÜTZMACHER, A.D.; GARCIA, M.S.; ZOTTI, M.J.; NÖRNBERG, S.D.; MAGALHÃES, T. R.; BANDEIRA, J.M. Susceptibilidade de lagartas dos 
biótipos milho e arroz de Spodoptera frugiperda (J.E. Smith, 1797) (Lepidoptera: Noctuidae) a inseticidas com diferentes modos de ação. Ciência Rural, v.36, n.1, p.15-20, 2006.

CARVALHO, G.A.; BEZERRA, D.; SOUZA, B.; CARAVLHO, F.C. Efeitos de inseticidas usados na cultura do algodoeiro sobre Chrysoperla externa (Hagen) (Neuroptera: Chrysopidae). Neotropical Entomology, v.32, n.3, p.699-706, 2003.

\section{CISNEROS, J.; GOULSON, D.; DERWENT, L.C.;} PENAGOS, D.I.; HERNANDÉZ, O.; WILLIANS, T. Toxic effects of spinosad on predatory insects. Biological Control, v.23, n.4, p.156-163, 2002.

FALEIRO, F.G.; PICANÇO, M.V.; De PAULA, S.V.; BATALHA, V.C. Seletividade de inseticidas a Spodoptera frugiperda (J.E. Smith) (Lepidoptera: Noctuidae) e ao predador Doru luteipes (Scudder) (Dermaptera: Forficulidade). Anais da Sociedade Entomológica do Brasil, v.24, n.2, p.247-252, 1995.

FERREIRA, D.F. Análises estatísticas por meio do Sisvar para Windows versão 4. 0. In: REUNIÃO ANUAL DA REGIÃO BRASILEIRA DA SOCIEDADE INTERNACIONAL DE BIOMETRIA, 45., 2000, São Carlos. Anais. São Carlos: UFSCar, 2000. p.255-258.

FRANÇA, F.H.; BRANCO, M.C. Controle pragas de hortaliças usando reguladores de crescimento de insetos. In. SIMPÓSIO ANUAL SOBRE O USO DE AGROTÓXICOS EM HORTALIÇAS, 14., 1996, Tinguá. Anais. Brasília: EMBRAPA: CNPH, 1996. p.4-8.

GRÜTZMACHER, A.D.; MARTINS, J.F. da S.; CUNHA, U.S. da Insetos-pragas das culturas do milho e sorgo no agroecossistema de várzea. In: PARFITT, J.M.B. (Ed.). Produção de milho e sorgo em várzea. Pelotas: Embrapa Clima Temperado, 2000. p.87-102.

HASSAN, S.A.; BIGLER, F.; BLAISINGER, P.; BOGENSCHÜTZ, H.; BRUN, J.; CHIVERTON, P.; DICKLER, E.; EASTERBROOK, M.A.; EDWARDS, P.J.; ENGLERT, W.D.; FIRTH, S.I.; HUANG, P.; INGLESFIELD, D.; KLINGAUF, F.; KÜHNER, C.; LEDIEU, M.S.; NATON, E.; OOMEN, P.A.; SHIRES, S.W.; STÄUBLI, A.; STEVENSON, J.; TUSET, J.J.; VANWETSWINKEL, G.; van ZON, A.Q.P. Standard methods to test the side effects of pesticides on natural enemies of insects and mites developped by the IOBC/ WPRS working group "Pesticides and beneficial organisms". OEPP/EPPO Bulletin, v.15, p.214-255, 1985.

LANGSTON, R.R.; POWELL, J. A. The earwigs of California (Order Dermaptera). Bulletin of the California Insect Survey, v.20, p.1-25, 1975.

MEDINA, P.; SMAGGHE, G.; BUDIA, F.; Del ESTAL, P.; TIRRY, L.; VIÑUELA, E. Significance of penetration, excretion and transovarial uptake to toxicity of three insetcs growth regulators in predatory lacewing adult. Archives of Insect Biochemistry and Physiology, v.51, n.2, p.91-101, 2002.

PASINI, A.; PARRA, J.R.P.; LOPES, J. Dieta artificial para criação de Doru luteipes (Scudder) (Dermaptera: Forficulidae), predador da lagarta-do-cartucho do milho, Spodoptera frugiperda (J.E. Smith) (Lepidoptera: Noctuidae). Neotropical Entomology, v.36, p.308-311, 2007.

PICANÇO, M.C.; De MOURA, M.F.; MIRANDA, M.M.M.; GONTIJO, L.M.; FERNANDES, F.L. Seletividade de inseticidas a Doru luteipes (Scudder, 1876) (Dermaptera: Forficulidae) e Cotesia sp. (Hymenoptera: Braconidae) inimigos naturais de Ascia monuste orseis (Godart, 1818) (Lepidoptera: Pieridae). Ciência Rural, v.33, n.2, p.183-188, 2003.

REIS, L.L.; OLIVEIRA, L.J.; CRUZ, I. Biologia e potencial de Doru luteipes no controle de Spodoptera frugiperda. Pesquisa Agropecuária Brasileira, v.23, n.4, p.333-342, 1988.

RETNAKARAM, A.; WRIGTH, J.E. Control of insects pests with benzoilphenyl ureas. In. WRIGTH, J.E.; RETNAKARAM, A. (Ed.). Chitin and bezoilphenyl ureas. Dordrecht: Dr Junk Publishing, 1978. p.205-282.

RETNAKARAM, A.; HIRUMA, K.; PALLI, S.R. Molecular analysis of the mode of action of RH-5992, a Lepidopteran-specific, non-steroidal ecdysteroid agonist. Insects Biochemistry and Molecular Biology, v.25, n.1, p.109-117, 1995.

SASAKI, E.T.; MUZETTI, E.J.P.; CALAFIORI, M.H. Controle de Spodoptera frugiperda (J.E. Smith, 1797) por tesourinha Doru lineare Eschs. Ecossistema, v.11, n.3, p.14-17, 1986.

SCOTT, A.J.; KNOTT, M.A. A cluster analyses method for grouping means in the analyses of variance. Biometrics, v.30, n.3, p.507-512, 1974.

SIMÕES, J.C.; CRUZ, I.; SALGADO, L.O. Seletividade de inseticidas às diferentes fases de desenvolvimento do predador Doru luteipes (Scudder) (Dermaptera: Forficulidade). Anais da Sociedade Entomológica do Brasil, v.27, n.2, p.289-294, 1998.

SOLTANI, N. Effects of ingested diflubenzuron on the longevity and the peritrophic membrane of adult mealworms (Tenebrio molitor L.). Pesticide Science, v.15, n.3, p.221-225, 1984.

STOCK, D.; HOLLOWAY, P.J. Possible mechanism for surfactant-induced foliar uptake of agrochemicals. Pesticide Science, v.38, n.2, p.165-177, 1993.

TANZUBIL, P.B.; McCAFFERY, A.R. Effects of azadirachtin and aqueous neem seed extracts on survival, growth and development of the African 
armyworm, Spodoptera exempta. Crop Protection, v.9, n.3, p.383-386, 1990.

VARGAS, E.R.; GARCIA, F.R.M.; KUSSLER. A.L. Seletividade de inseticidas utilizados no controle de Spodoptera frugiperda a adultos de D. luteipes. In. CONGRESSO BRASILEIRO DE ENTOMOLOGIA, 20., 2004, Gramado. Livro de resumos. Bento Gonçalves: 2004. n.317, p.341.
ZOTTI, M.J.; GRÜTZMACHER, A.D.; GRÜTZMACHER, D.D.; DALMAZZO, G.O.; MARTINS, J.F.S. Seletividade de inseticidas usados na cultura do milho para ninfas e adultos de D. lineare (Eschscholtz, 1822) (Dermaptera: Forficulidae) em semi-campo. Revista Brasileira de Agrociência, v.14, n.3, p.317-325, 2008.

Recebido em 3/3/09

Aceito em 23/5/10 